\title{
Pure left hippocampal stroke: a transient global amnesia-plus syndrome
}

\author{
Antonio Carota $\cdot$ Andreas P. Lysandropoulos • \\ Pasquale Calabrese
}

Received: 5 August 2011/Revised: 12 October 2011 / Accepted: 15 October 2011/Published online: 29 October 2011 (c) Springer-Verlag 2011

Dear Sirs,

Human and animal studies have provided undeniable evidence that the hippocampus is critically involved in supporting declarative-recollective memory [1,2]. Isolated unilateral first-ever ischemic stroke that is specifically confined to the hippocampal region is an extremely rare event $[3,4]$ and similar cases have not generally been studied with standardized neuropsychological assessment in the acute phase.

A 41-year-old right-handed office worker presented abruptly with a dense amnesic syndrome (both anterograde and retrograde memory deficit) resembling transient global amnesia (TGA). Knowledge of personal identity was maintained, other cognitive domains were spared and he repeatedly asked questions such as "where am I?, what are we doing?". However, differently than what is generally expected with TGA, the patient had frequent anomic pauses in spontaneous speech and committed significant errors in naming tests, mostly omissions ("amnesic aphasia").

Brain MRI at day 3 after admission (Fig. 1) showed a unique acute infarction of the dorsal part of the left hippocampal body, most likely explained by occlusion of the

\footnotetext{
A. Carota $(\square)$

Hildebrand Clinic, Rehabilitation Center,

6614 Brissago, Switzerland

e-mail: a.carota@bluewin.ch
}

A. Carota - A. P. Lysandropoulos

Neurology Service, CHUV, Lausanne, Switzerland

P. Calabrese

Department of Psychology, University of Basel,

Basel, Switzerland

P. Calabrese

Neurocenter of Southern Switzerland, Lugano, Switzerland most distal PCA branches (middle or posterior hippocampal artery as suggested in [3]). The etiology was a large patent foramen ovale as other disorders were not identified.

The amnesic syndrome resolved within $24-48 \mathrm{~h}$ (including retrograde amnesia) but verbal anterograde memory deficits persisted over 4 weeks and afterwards normalized. The neuropsychological assessment was performed within the first week after stroke. The patient performances were in the norms in the following domains: attention (WAIS code, TMT-A, TAP), immediate memory (Hebb and Corsi test), calculation (Barcelona battery), gestural praxis (Florida Apraxia Battery), visuo-perceptive attitudes (Rey-complex figure copy, bisection and cancelation tasks, face recognition, Hooper test), executive functions (FAB, WCST, BADS, Ruff Figural Fluency, TMT B, color stroop), reasoning (SPM47), retrograde memory (autobiographical memory interview). Language (Monreal Toulouse Protocol) was normal except for mild anomia and reduced literal fluencies. There was a dissociation of performance between verbal (forward 4, backward 3) and visual (forward 7, backward 6) span tests.

Verbal learning was assessed through the Rey auditory verbal learning test (15 words), nonverbal learning by means of the Rey visual design learning test ( 15 designs), the recall phase of the Rey-complex figure (RCF) and recognition memory test for words and faces. In the verbal modality, free recall (sum of five trials: 27,30 -min delayed recall: 1) and delayed recognition (score: 4 ) were impaired, while the same domains were within normal limits in the visual modality (sum of five trials: 45,30 -min recall: 11 , recognition: 13) (Fig. 2a). Intrusions (sum of five trials: 28) and errors in recognition (score: 10) of the learning tests were limited to the verbal modality (Fig. 2b). The results of the RCF immediate and delayed recall were in the normal range (40 and 50 percentile). 

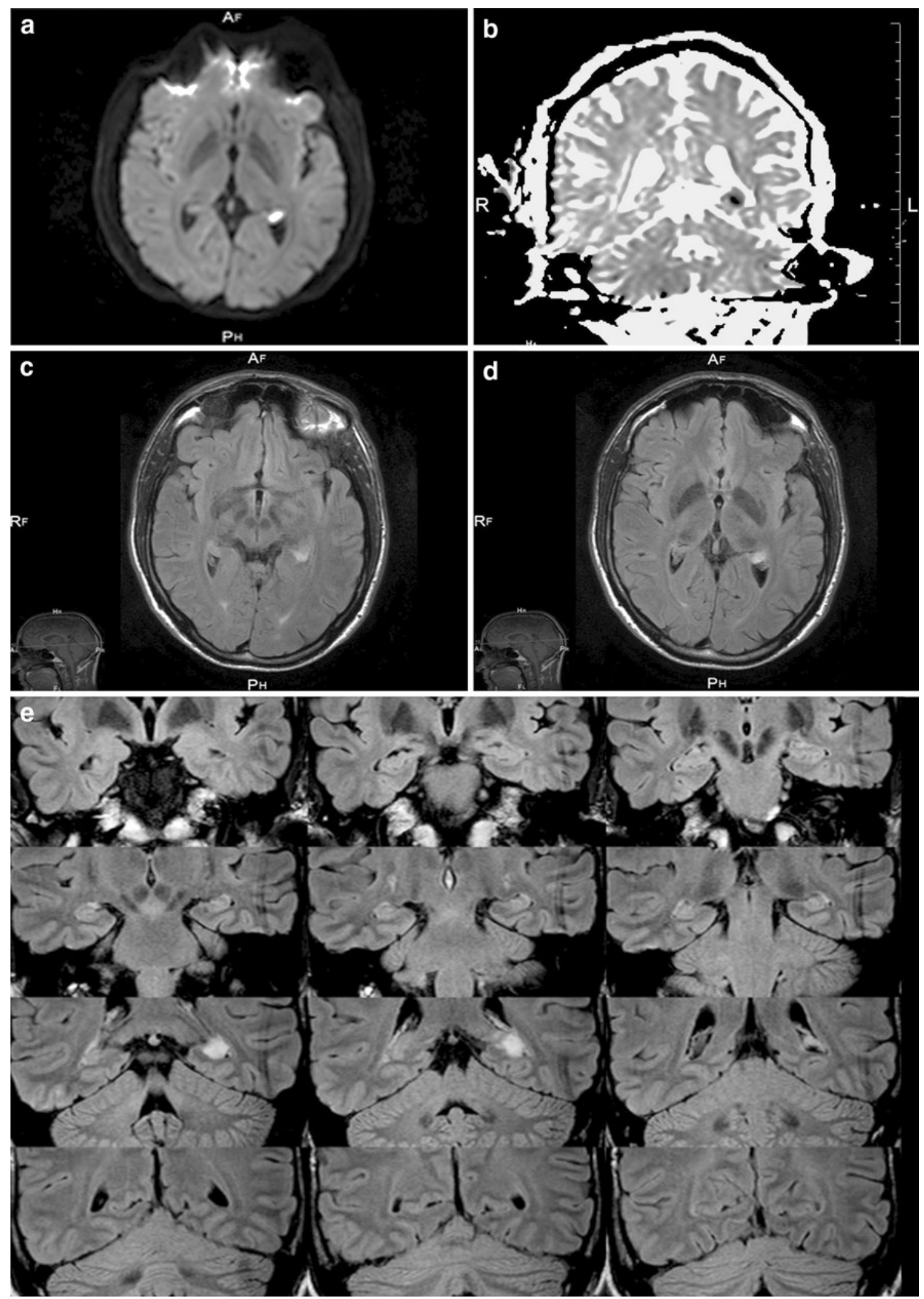

Fig. 1 Axial DWI (a), coronal ADC (b), axial FLAIR MRI images (c, d) showing abnormal signal (suggesting acute stroke) in the left dorsolateral hippocampal body. e FLAIR MRI coronal sections (anteroposterior direction going from the left of the figure to the right) showing the recent ischemic lesion in the dorsal and lateral part of the left hippocampal body 
Fig. 2 Patient's performances in verbal and visual memory tests. Part a reports the percentile scores and $\mathbf{b}$ the number of intrusions or false recognition for verbal

(15 words) and visual (15 signs) learning. Part $\mathbf{c}$ reports the percentage of correct responses and false recognitions in Warrington recognition memory test for words and faces a

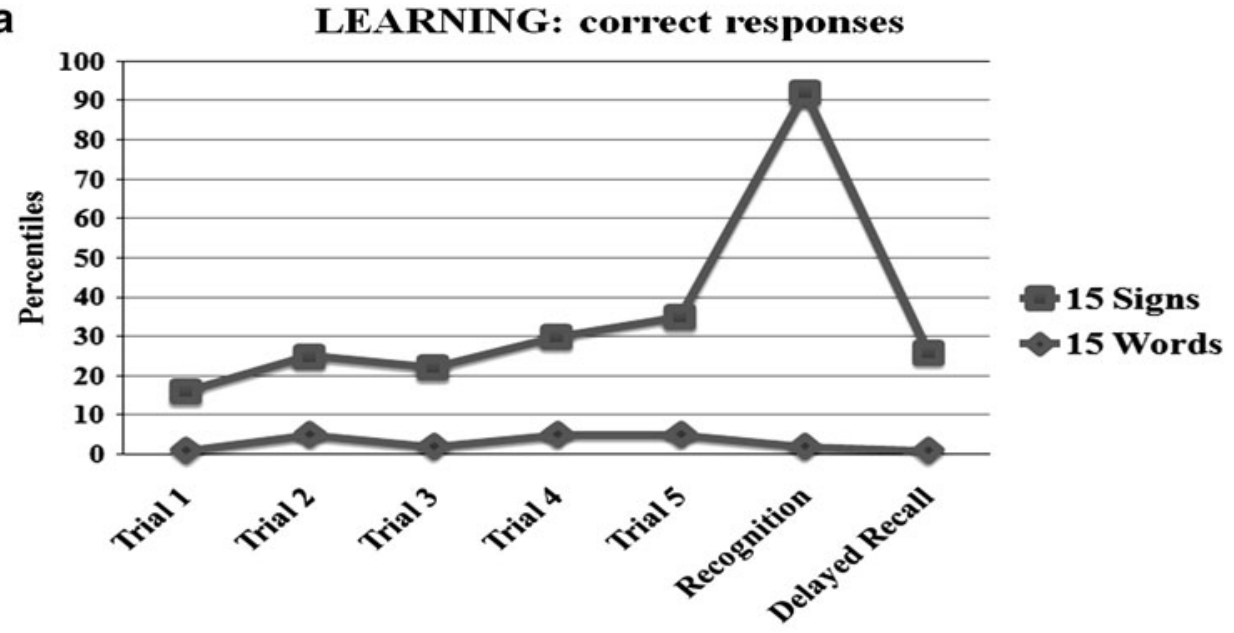

b

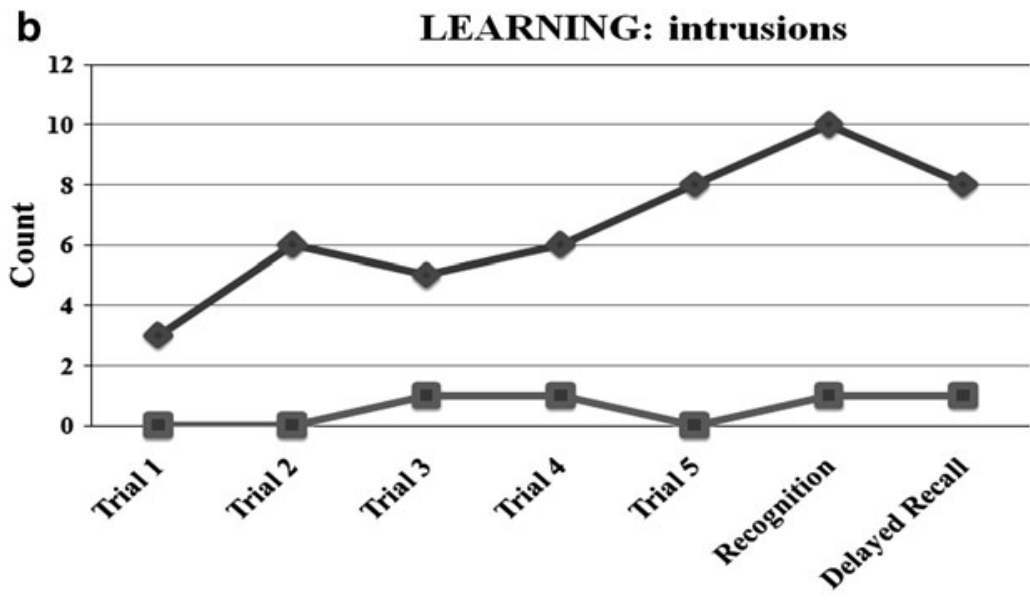

15 Words 15 Signs
C

\section{RECOGNITION of recurrent stimuli}

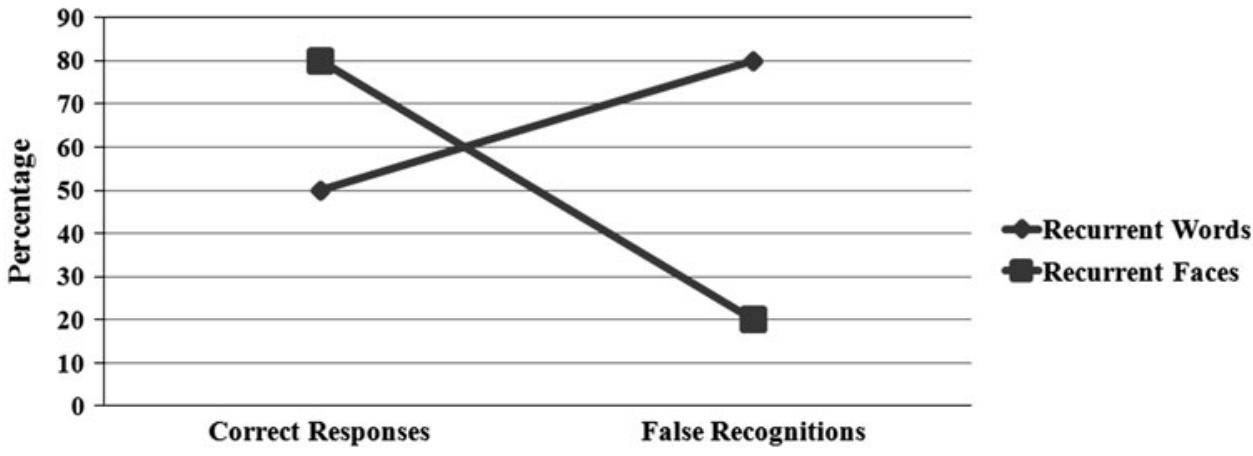

A significant dissociation was found between performances of verbal (recognition: 20/40, false recognition: 35) and visual (recognition: 33/40; false recognition: 8) modalities with the recognition memory test for words and faces (Fig. 2c).

A neuropsychological assessment was performed 3 weeks later after the first examination and showed complete normalization of performances in verbal memory tests and language.
The main finding of this report is that unilateral pure unique ischemic stroke confined to the mediodorsal region of the hippocampus manifests in the acute phase with a cognitive profile that is quite similar to transient global amnesia (TGA) except for "amnestic" or "anomic aphasia" (TGA-plus syndrome). Lesion localization are similar to the findings of those MRI-DWI [5, 6] or MRI spectroscopy studies [7] of patients with TGA that showed delayed hippocampal focal changes, which were limited 
to the lateral and/or dorsal region of the hippocampal body .

Our findings indicate the occurrence of anterograde memory deficits, as the typical features of medial temporal lobe dysfunction (encoding deficits, semantic intrusions, and lack of primacy effect). Hence, they provide further evidence for the specialization of the left hippocampus for phonological working memory, verbal encoding and recollection $[3,4]$ and for lexical retrieval in naming tasks [8-10].

As suggested by similar reports [3], memory deficits appear to be transitory for unilateral hippocampal stroke.

Conflict of interest None.

\section{References}

1. Eichenbaum H, Yonelinas AP, Ranganath C (2007) The medial temporal lobe and recognition memory. Ann Rev Neurosci 30:123-152

2. Squire LR, Wixted JT, Clark RE (2007) Recognition memory and the medial temporal lobe: a new perspective. Nat Rev Neurosci 8(11):872-888
3. Szabo K, Förster A, Jäger T, Kern R, Griebe M, Hennerici MG, Gass A (2009) Hippocampal lesion patterns in acute posterior cerebral artery stroke: clinical and MRI findings. Stroke 40(6): 2042-2045

4. Ott BR, Saver JL (1993) Unilateral amnesic stroke. Six new cases and a review of the literature. Stroke 24:1033-1042

5. Enzinger C, Thimary F, Kapeller P, Ropele S, Schmidt R, Ebner F, Fazekas F (2008) Transient global amnesia: diffusion-weighted imaging lesions and cerebrovascular disease. Stroke 39(8): 2219-2225

6. Sedlaczek O, Hirsch JG, Grips E, Peters CN, Gass A, Wöhrle J, Hennerici M (2004) Detection of delayed focal MR changes in the lateral hippocampus in transient global amnesia. Neurology 66(12):2165-2170

7. Bartsch T, Alfke K, Deuschl G, Jansen O (2007) Evolution of hippocampal CA-1 diffusion lesions in transient global amnesia. Ann Neurol 62(5):475-480

8. Davies KG, Bell BD, Bush AJ, Hermann BP, Dohan FC, Jaap AS (1998) Naming decline after left anterior temporal lobectomy correlates with pathological status of resected hippocampus. Epilepsia 39(4):407-419

9. Menke R, Meinzer M, Kugel H et al (2009) Imaging short- and long-term training success in chronic aphasia. BMC Neurosci 10:118

10. Hamberger MJ, Seidel WT, McKhann GM, Goodman RR (2010) Hippocampal removal affects visual but not auditory naming. Neurology 74(19):1488-1493 